\title{
Fixed Broadband Wireless Access (FBWA) with Practical OFDM System
}

\author{
Lokesh C., Dr. Nataraj K. R., Dr. Rekha K. R . \\ ${ }^{1}$ Assistant Professor,Department of E \& E E.VVCE,Mysore, India. \\ $2 \& 3$ Professor, Department of E \& C E, SJBIT, Bangalore, India.
}

\begin{abstract}
It has been widely recognized that fixed broadband wireless systems are a viable alternative to overcoming the "last mile" distribution problem of bringing high speed data connections to the end user. In areas where the cellular coverage and wired infrastructure are inadequate or nonexistent, fixed wireless solutions can be deployed much more quickly and at a fraction of the cost compared to wired networks. There are currently several solutions being deployed or proposed to address this emerging fixed broadband wireless market. These solutions can be categorized based on various parameters including frequency band, range, bandwidth, throughput, regulatory and licensing requirements, and the like. The Local Multipoint Distribution Service has extremely high bandwidth; however, due to short range and LOS coverage limitations, it is not economically feasible for deployment for other than highly populated urban areas. The Multichannel Multipoint Distribution System, with its longer range, is a better match to cover medium-density areas, but it requires payment of a licensing fee. The unlicensed spectrum allows for cost-effective immediate deployment and is attractive to unlicensed operators with limited financial resources.
\end{abstract}

Index Terms: - Multichannel Multipoint Distribution System (MMDS), Local Multipoint Distribution Service (LMDS), Code Division Multiple Access(CDMA), Time Division Multiple Access(TDMA), Frequency Division Multiple Access(FDMA), Message Authentication Code(MAC).

\section{INTRODUCTION}

At present wire line and cellular systems are suffering from a capacity and bandwidth crunch due to various economic, legal, and political causes $[1,2]$. The delay in the deployment of $3 \mathrm{G}$ systems makes the situation even worse. Various technologies are being developed to fill the void of "last mile/hotspot" coverage based on fixed broadband wireless technology. These networks are typically configured in a cell based point-tomultipoint topology. The deployment of these networks requires a high initial up-front investment to pay for base stations and backbone networks. Additionally, a significant number of potential subscribers may not be reached due to LOS constraints. This chapter proposes a solution based on OFDM wireless communication techniques to resolve the issue of coverage and capacity. The solution is based on proven technology and an offshoot of existing standards so that it is cost effective to implement without much of an upfront cost in engineering and production.

The proposal utilizes the existing IEEE 802.16 standard for Wireless MAN ${ }^{\mathrm{TM}}$ MAC and higher-layer interfaces to minimize non-PHY development costs [3, 4]. The IEEE standards committee has recently standardized the IEEE 802.16 Wireless MAN air interface based on OFDM techniques to serve the needs of the BWA scheme primarily to build a metropolitan point-to-multipoint communications infrastructure. The air interface is designed to carry any type of data or multimedia traffic with full QoS support. The MAC supports burst FDD and TDD in a consistent framework. The application of OFDM technology in wireless applications has continued to grow and has become the forerunner for the next generation of cellular/wireless systems across the globe. The requirements for the PHY of the proposed local coverage are different from those proposed in the IEEE 802.16 standard [5].

\section{CELL-BASED INFRASTRUCTURE}

A large geographical area is covered using the cell-based infrastructure. One base station covers a predefined geographical area and handles the communications needs of the subscribers in that area of coverage. The non-over lapping base stations are duplicated at regularly spaced intervals to provide wider coverage area with the frequency reuse principle. These base stations employ various medium-access mechanisms to coordinate and maintain the subscribers' communication links. This medium-access scheme can be based on TDMA, FDMA, CDMA or OFDM techniques. The base station provides controlled access to bandwidth allocation and sharing between subscribers. 


\section{A. Coverage Difficulties in Cell-Based Infrastructure:}

The disadvantage of the cellular system is that it requires a central location (like a mobile switching center for GSM/GPRS and UMTS, and so forth) through which all of the traffic must be channeled. This puts an unnecessary burden on the core and backbone networks because they need to be deployed for the maximum capacity envisioned [6]. The system does not allow for scalability or provide an easy migration path for growth.

Table 1 : Fixed Broadband Wireless Technology

\begin{tabular}{|c|c|c|c|}
\hline Type & Frequency band (GHz) & Bandwidth & Coverage(miles) \\
\hline LDMS & 28 & $1.2 \mathrm{GHz}$ & 3 \\
\hline MMDS & 2.5 & $200 \mathrm{MHz}$ & 30 \\
\hline ISM(Unlicensed) & 2.4 & $83 \mathrm{MHz}$ & 30 \\
\hline UNII (Unlicensed) & 5.8 & $200 \mathrm{MHz}$ & 12 \\
\hline
\end{tabular}

The cellular system also experiences coverage outages due to shadowing (loss of LOS) in high-rise office/residential and hilly areas. This has been overcome in certain circumstances by using smaller cells to provide coverage in fringe areas, but this adds costs for additional base stations and backbone networks.

\section{B. Mesh Topology-Based Infrastructure}

To provide the coverage in non-LOS (NLOS) areas, a "mesh" network topology has been employed. In this topology, each station is equipped with an Omni directional antenna [7]. Each station belonging to a network is capable of sending, receiving, and forwarding packets from any of its neighbors to various destinations using any of the possible routes. This topology does not require a backbone and can easily reach hidden locations through multiple hops.

There are some distinct disadvantages of this multipoint-to-multipoint network connectivity:

- The antenna is typically Omni directional to cover the neighboring stations, which either reduces the range or decreases the power efficiency of the station.

- Each station has a good number of neighboring stations that can be reached with one hop. Although this is an advantage of mesh topology, which provides many alternate paths between any two points, the packet will reach not only the intended recipient, but all other unintended stations as well, possibly causing collisions.

- To avoid such collisions, there is a need for over-the-air coordination of packet routing of information, adding extra overhead to the packet. This distribution overhead reduces the achievable throughput of the system [8].

\section{PROPOSED FBWA}

The proposed solution is based on point-to-multipoint communications employing a mesh topology, which takes advantage of both cellular and mesh network topologies, but avoids the pitfalls of both. The proposed solution consists of a network topology with MAC protocol that facilitates the following:

- The network can be deployed incrementally as the need for coverage arises, thus providing scalability and an easy migration path to growth.

- The stations utilize the configurable directional (beam-forming) antenna, the width and direction of which can be configured through software control [9].

- Any new station can be added to the overall network infrastructure with easy coverage computation and link upgrade.

- Frequency and space diversity can be added to enhance the communication link and channel capacity if required.

- All the nodes in the network employ similar equipment.

- No expensive hubs or base stations are required.

- Backbone point-to-point links can be added later when a need for network expansion arises.

\section{SYSTEMS REQUIREMENTS}

This section specifies the requirements based on the proposed single-hop point-to multipoint (SHPM) network topology for use in the FBWA application. The PHY shall provide digital, two-way voice, data, Internet, and multimedia services. It shall be compatible with traditional wire line, cable, DSL, and T1/E1 services.

To maximize the efficient utilization of spectrum in the unlicensed band, the air interface supports the multiplexing of subscribers using TDMA technology [10]. The main features of the proposed PHY are as following: 
- Duplex mode of operation (simultaneous Tx/Rx) using TDD;

- Full compatibility with IEEE 802.16 MAC;

- Uplink multiple access using TDMA;

- Downlink multiple access using broadcasts TDM;

- Adaptive modulation and FEC coding in both up- and downlink;

- Support for smart antenna;

- High-speed asymmetric data-rate services;

- Dynamic allocation of bandwidth/channel;

- Allowance for handover and limited roaming;

- Easily adoption of cellular/wire line infrastructure.

This section primarily deals with the PHY requirements. The OFDM parameters are chosen based on these requirements. The systems PHY requirements are depicted in tabular form in Table 2.

Table 2: System PHY Parameters.

\begin{tabular}{|l|l|l|}
\hline \multicolumn{1}{|c|}{ Parameters } & \multicolumn{1}{c|}{ Value } & \multicolumn{1}{c|}{ Comments } \\
\hline Frequency bands & $5.725-5.825 \mathrm{GHz}$ & UNII base total BW $=100 \mathrm{MHz}$ \\
\hline Coverage range & $\leq 10$ miles & Last mile hotspot coverage \\
\hline Delay spread tolerance & $\leq 10 \mu \mathrm{s}$ & Urban area \\
\hline Channel BW & $6 \mathrm{MHz}$ & 16 channel with end guard bands of $2 \mathrm{MHz}$ \\
\hline Data rate & $2,4,8,12 \mathrm{Mbps}$ & \\
\hline
\end{tabular}

\section{PARAMETER SELECTION}

Based on the PHY requirements depicted in Table 8.2, OFDM-based systems parameter lists have been selected to fulfill the systems PHY requirements.

- Selection of Guard Period: The OFDM-based fixed broadband wireless system needs to tolerate an RDS of about $10 \mu$ s. The guard period of $40 \mu \mathrm{s}$ (four times the RDS) is chosen to mitigate any losses due to ISI.

- Selection of OFDM Symbol Period: It is desirable to reduce to a minimum the throughput loss due to the addition of a guard period; therefore, a symbol period five to six times larger than the guard period is chosen. An OFDM symbol period of $240 \mu$ s (six times the guard period) is chosen, which results in less than 1-dB loss in throughput[11].

- Selection of SC Spacing: Number of SCs, Modulation, and Coding Rate Based on the guard period and OFDM symbol period, the spacing of SCs is computed so that they are orthogonal to each other. The SC spacing is computed as the inverse of the useful OFDM symbol period (1/(OFDM symbol period - guard period $)=1 /(240-40) \mu \mathrm{s}=5 \mathrm{kHz})[12]$.

The total channel bandwidth is allocated to $6 \mathrm{MHz}$ and the parameter choice is tabularized in Table 3 .

Table 3: BWAS parameters list

\begin{tabular}{|c|c|c|c|c|}
\hline Parameter & \multicolumn{4}{|c|}{ Number of SCs, 1,024; SCs used, 960 } \\
\hline Data rate & $2 \mathrm{Mbps}$ & $4 \mathrm{Mbps}$ & $8 \mathrm{Mbps}$ & $12 \mathrm{Mbps}$ \\
\hline Coding rate & $1 / 2$ & $1 / 2$ & $3 / 4$ & $2 / 3$ \\
\hline Mapping/Modulation & BPSK & QPSK & $16-$ QAM & $64-$ QAM \\
\hline Number of pilot insertion & 16 & 16 & 16 & 16 \\
\hline
\end{tabular}

\section{CONCLUSION}

In This The fixed BWAS based on the OFDM scheme was proposed and requirements were set to complement the wire line and wireless infrastructure in last-mile and hotspot coverage to provide a low-cost, quickly deployable solution. The proposed systems were designed; the PHY parameters and the access techniques for uplink and downlink transmission were formulated based on the OFDM scheme. The concept of a point-to-multipoint mesh network topology was proposed to mitigate the NLOS situation, and the requirements for central, parent, and child site transceiver antennas were set. The choice of beam-forming antenna under software control allowed the promotion of any node to a parent node and enabled nodes to serve as hop or relay agents for neighboring nodes without direct LOS to the central station. 


\section{REFERENCES}

[1] Jha, U., "Wireless Landscape-A Need for Seamless Connectivity," Wireless Personal Communication (WIRE) Journal, Vol. 22, No. 2, August 2002, pp. 275-283.

[2] Jha, U. S., et al., "Novel Application-Specific Signal Processing Architectures for Wideband CDMA and TDMA Applications," VTC 2000, Tokyo, Japan, 2000.

[3] IEEE802.16.3-00/02r4, Functional Requirements for the 802.16.3 Interoperability Standard, dated 200009-22.

[4] Porter J. W., and J. A. Thweatt, "Microwave Propagation Characteristics in the MMDS Frequency Band," Proc. Intl. Conf. on Communications, New Orleans, LA, June 2000, pp. 1578 -1582.

[5] Sari, H., G. Karam, and I. Jeanclaude, "Transmission Techniques for Digital Terrestrial TV Broadcasting," IEEE Communications Magazine, Vol. 33, No. 2, February 1995, 100-109.

[6] Tarokh, V., and H. Jafarkhani, "On the Computation and Reduction of the Peak-to- Average Ratio in Multicarrier Communications," IEEE Trans. on Communications, Vol. 48, No. 1, January 2000, pp. 3744.

[7] IEEE 802.16ab-01/01rl, Air Interface for Fixed Broadband Wireless Access Systems, Part A: Systems between $2-11 \mathrm{GHz}$, July 2001.

[8] ISO 15802-3:1998, Information Technology-Telecommunications and Information Exchange between Systems-Local and Metropolitan Area Networks-Common Specifications- Part 3: Media Access Control (MAC) Bridging.

[9] ISO/IEC 15802-5:1998, Information Technology-Telecommunications and Information Exchange between Systems - Local and Metropolitan Area Networks - Common Specifications - Part 5: Remote Media Access Control (MAC) Bridging.

[10] IEEE 802. IF-1993, IEEE Standards for Local and Metropolitan Area Networks: Common Definitions and Procedures for IEEE 802 Management Information.

[11] LAN/MAN Standards Committee of the IEEE Computer Society, Part 11: Wireless LAN Medium Access Control (MAC) and Physical Layer (PHY) Specifications-High Speed Physical Layer in the $5 \mathrm{GHz}$ Band, ANSI/IEEE Std 802.11, 1999 Edition.

[12] Jha, U., "Low Complexity Resource Efficient OFDM Transceiver Design," ICPWC-2002, New Delhi, India, December 15-18, 2002. 